\title{
Are CT and MRI scanning reliable in diagnosing otogenic skull base osteomyelitis in non- immunocompromised elderly patients? a case series and review of the literature
}

\begin{abstract}
Background: Otogenic skull base osteomyelitis (SBO) is a life-threatening condition that requires timely diagnosis and treatment. It is most commonly seen in the elderly diabetic or immunocompromised patients. The role of nuclear medicine imaging in diagnosis of SBO remains controversial.

Methods: This paper presents two unusual cases of otogenic SBO in elderly nonimmunocompromised patients. A review of the relevant literature from 1986 to 2016 was performed via the Medline database using search terms 'skull base osteomyelitis', 'otogenic', 'malignant otitis externa' and 'imaging'.

Results: Both patients presented with chronic mild otalgia and ear discharge, and were subsequently diagnosed with otogenic SBO based on nuclear medicine imaging. Nuclear medicine imaging was essential for diagnosis and localization of SBO in both patients as CT and MRI either failed to detect or accurately localize SBO.

Conclusion: SBO can occur in non-immunocompromised elderly patients with normal CT and MRI scans. Nuclear medicine imaging is essential in the diagnostic workup.

Keywords: skull base osteomyelitis, nuclear medicine, bone scan, computed tomography, magnetic resonance imaging, otogenic, single-photon emission computed tomography
\end{abstract}

\author{
Volume 3 Issue 5 - 2017

\begin{abstract}
Rebecca SM Lim,' Chi-kee Leslie Shaw ${ }^{1,2}$
'Department of Otolaryngology and Head \& Neck Surgery, Modbury Hospital,Australia
\end{abstract} \\ ${ }^{2}$ Discipline of surgery, University of Adelaide, Australia
}

\begin{abstract}
Correspondence: Rebecca Lim, Department of Otolaryngology and Head \& Neck Surgery, Modbury Hospital, Adelaide, South Australia, Email rebecca I 188@gmail.com
\end{abstract}

Received: June 05, 2017 | Published: July 26, 2017
Abbreviations: SBO, skull base osteomyelitis; CT, computed tomography; MRI, magnetic resonance imaging; CRP, c-reactive protein; ESR, erythrocyte sedimentation rate; SPECT-CT, single-photon emission computed tomography-computed tomography

\section{Introduction}

Otogenic skull base osteomyelitis (SBO) is a potentially lifethreatening condition that requires timely diagnosis and treatment. It is most commonly seen in the elderly diabetic or immunocompromised patients. However, it can also occur in non-immunocompromised patients without diabetes. In these cases, a high index of suspicion on the part of the clinician is required in order for timely diagnosis. The diagnosis of SBO is made by a constellation of symptoms and signs, and typical radiological features on computed tomography (CT) or magnetic resonance imaging (MRI). The role of nuclear medicine scanning in the diagnosis of SBO remains controversial. ${ }^{1}$ We present two unusual cases of healthy, non-immunocompromised elderly patients with otogenic skull base osteomyelitis which were unable to be detected or accurately localized by a combination of CT and MRI.

\section{Case I}

An 89year-old gentleman with a medical history of atrial fibrillation, hypertension and hypothyroidism presented to an Otolaryngologist with a 2 month history of right-sided mild otalgia, ear discharge and hearing loss without cranial nerve palsy. He was treated with two courses of Augmentin Duo Forte and underwent an
MRI. This showed complete opacification of the petromastoid and middle ear (Figure 1A). Having had no improvement, he consulted a second Otolaryngologist, who found a large aural polyp arising from the middle ear and protruding laterally through a large tympanic membrane perforation. The ear swab grew Pseudomonas aeruginosa, sensitive to Tazocin. Blood tests revealed a normal white and red cell count, although a lymphocytosis was present. His neutrophil count and fasting glucose were normal. He did however have a slightly raised C-reactive protein (CRP) of $64 \mathrm{mg} / 1$ and a slightly elevated erythrocyte sedimentation rate (ESR) of $78 \mathrm{~mm} / \mathrm{h}$.

The patient was commenced on a further course of Augmentin Duo Forte and Ciproxin HC ear drops, but failed to improve. ACT temporal bone performed showed evidence of skull base osteomyelitis with demineralization involving the tegmen tympani, mastoid air cells and involving the temporal bone in the region of the pharyngotympanic tube (Figure 1B).

He then underwent a ${ }^{99 \mathrm{~m}} \mathrm{Tc}$ Single-photon emission computed tomography-computed tomography (SPECT-CT) hybrid scan. This revealed localized uptake in the right greater sphenoid wing, with mild activity in the right mastoid process (Figure 1C). 48hours later, these findings were confirmed on a SPECT-CT with 67-Gallium citrate. The patient was then treated with intravenous Tazocin ${ }^{\circledR}$ for a total of 4weeks and underwent hyperbaric oxygen therapy for 1week, with significant improvement in his symptoms. A Gallium SPECTCT study performed 2 weeks after the initial study showed stable osteomyelitis of the greater wing of the right sphenoid bone. 


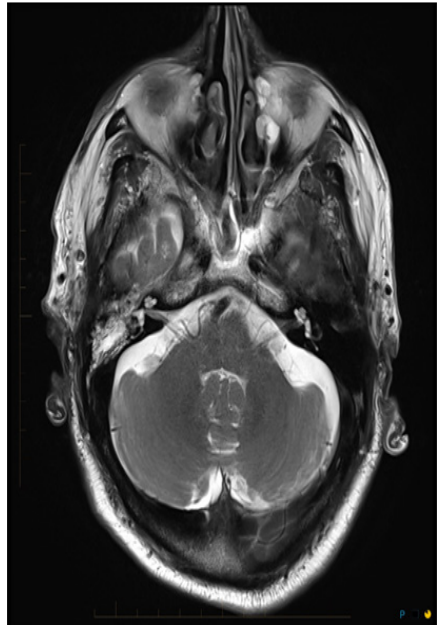

Figure IA Axial slice of T2, diffusion-weighted MRI showing soft tissue and fluid opacification within the right petromastoid air cells.

\section{Case 2}

A 95year-old lady presented with a 4week history of left mild otalgia and ear discharge which had not improved despite three courses of Augmentin Duo Forte and Soframycin ear drops. She was a restively fit non-diabetic who had not had any ear infections or surgery previously. On clinical examination, her left ear canal was completely occluded by a large aural polyp and the tympanic membrane could not be visualized. No cranial nerve abnormality was detected. A left ear swab isolated Pseudomonas aeruginosa sensitive to Tazocin. A microtoilet of her left ear was performed and she was treated with a further course of Augmentin Duo Forte and Ciproxin HC ear drops. Two weeks later, there was a marked reduction in size of the aural polyp and it was seen to be arising from the anterior wall of the ear canal. The tympanic membrane was still unable to be visualized.

A CT scan was then performed, which revealed a healthy middle

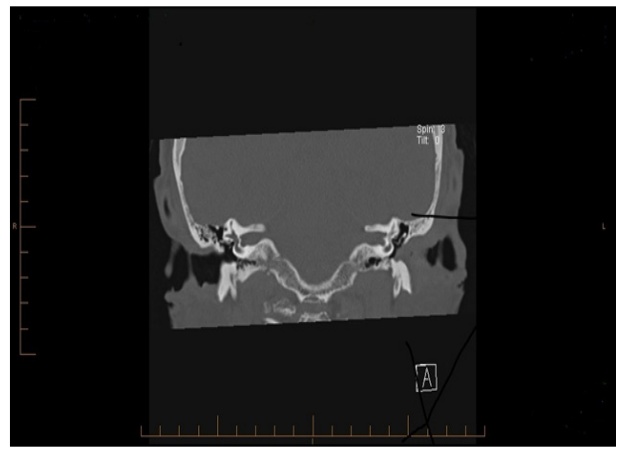

Figure 2A Coronal slice of CT petrous temporal bones showing left ear canal completely occluded by large soft tissue mass without bony destruction.

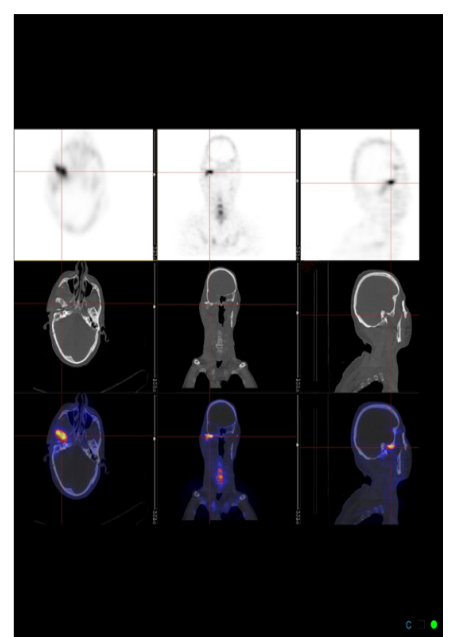

Figure IC SPECT-CT showing osteomyelitis in the right greater wing of sphenoid.
Figure IB Coronal CT slice showing evidence of skull base osteomyelitis with demineralization involving the tegmen tympani (green arrow). ear and mastoid, with no sign of skull base osteomyelitis (Figure 2A). The residual aural polyp was biopsied and found to be a benign inflammatory polyp. On subsequent review, the patient complained of soreness of the muscles of mastication and this was thought to be due to a temporomandibular joint disorder. An MRI scan was performed, which showed no TMJ pathology but revealed fluid in her left mastoid (Figure 2B). Blood tests including a full blood count and C-reactive protein were normal.

Despite having no risk factors apart from advanced age, a high index of suspicion for skull base osteomyelitis led to the patient undergoing a ${ }^{99 \mathrm{~m}}$ Tc SPECT-CT which revealed low grade tracer uptake in the left petrous temporal bone (Figure 2C). This was confirmed on a gallium SPECT-CT. The patient was then treated with intravenous Tazocin ${ }^{\circledR}$ for a total of 6 weeks followed by 3 months of dicloxacillin $1 \mathrm{~g}$ q.i.d and oral ciprofloxacin 500mg b.d.

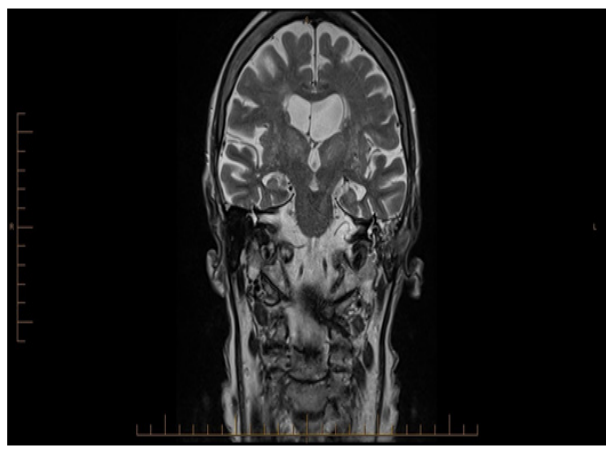

Figure 2B Coronal slice of T2-weighted MRI brain showing fluid in the left mastoid.

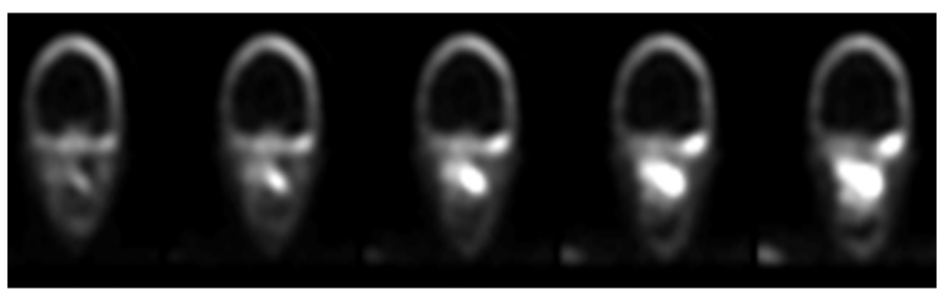

Figure 2C Gallium scan with SPECT showing increased bony uptake in the left petrous temporal bone. 


\section{Discussion}

Otogenic skull base osteomyelitis is a condition with significant morbidity and mortality if not treated appropriately. ${ }^{2}$ This condition typically affects elderly diabetic patients or those who are immunocompromised. ${ }^{3,4}$ Diabetes mellitus is thought to be a risk factor due to microvascular disease, especially in the external auditory canal, and its immunocompromising impact., ${ }^{4,5}$ Diabetes mellitus also leads to defects in chemotaxis and phagocytosis of macrophages and decreases the oxidative burst and killing function of polymorphonuclear leukocytes. $^{2}$

Although not definitively established in the current literature, it has been suggested that any conditions that adversely affect blood flow and oxygen delivery through bone, including malignancy, osteoporosis, Paget disease, anemia and radiation may be predisposing factors. ${ }^{6,7}$ The elderly are at a greater risk of developing SBO, possibly due to immunosenescence-the deterioration of their immune systems with age. ${ }^{8}$ The mechanisms behind these age-related defects are broad and range from defects in haemopoiesis to defects in peripheral lymphocyte migration, maturation and function. ${ }^{8-10}$

The clinical presentation of SBO can vary greatly, but patients tend to complain of severe, unremitting, throbbing otalgia., ${ }^{3,5}$ Cranial nerve palsies can occur and have been reported in close to half the patients in some series. ${ }^{3,5,11}$ Cranial nerve VII is the most commonly affected but cranial nerves IX, X, XI and XII can also be involved. ${ }^{12}$ This is thought to occur due to the infection traversing the fissures of Santorini and the tympanomastoid suture to the Haversian system of the compact bone, and finally to the skull base. ${ }^{2,13}$

The initial workup of suspected SBO should include a complete blood count and inflammatory markers such as ESR and CRP. An elevated ESR might help distinguish SBO from a malignant process. ${ }^{2}$ Taking a swab of the ear discharge is always useful for identifying the organism responsible which ultimately influences antimicrobial therapy. Pseudomonas and Staph aureus are the two most common bacterial organisms that cause $\mathrm{SBO} .^{2}$ The diagnosis of $\mathrm{SBO}$ can be challenging as $\mathrm{CT}$ scans may not detect early destructive changes in the cortical bone. In fact, it has been suggested that trabecular bone erosion of between $30-50 \%$ is necessary to give a positive CT scan. ${ }^{14,15}$

While MRI is unable to detect bony destruction, it excels at characterizing the soft tissue planes at the skull base. This is especially useful in patients with cranial nerve palsies secondary to SBO. ${ }^{16,17}$ Most abnormalities on MRI exhibit a low signal on T1weight images and a high signal on T2-weighted images, indicating replacement of the normal fatty marrow. ${ }^{18}$ However, differentiating a skull base malignancy from SBO on MRI can be challenging. ${ }^{19}$ Given that neither test is diagnostic, most studies recommend a CT and MRI together in order to obtain as much information as possible. ${ }^{13,16,17,20}$

Another imaging option is a radionucleotide scan, including ${ }^{99 \mathrm{~m}} \mathrm{Tc}$ or gallium- 67 bone scans. Though not standard practice at present, these imaging modalities have some advantages over CT and MRI. The ${ }^{99 \mathrm{~m}} \mathrm{Tc}$ radiotracer accumulates in areas of osteoblastic activity, and has up to $100 \%$ sensitivity to osteomyelitis. ${ }^{21}$ When combined with SPECT-CT, the sensitivity and disease localization of the scan is improved, possibly offering a higher accuracy in detecting skull base osteomyelitis than $\mathrm{CT}$ alone. ${ }^{19}$ However, ${ }^{99 \mathrm{~m}} \mathrm{Tc}$ bone scans are relatively non-specific as there have been reports of positive scans in patients with simple otitis externa. ${ }^{21}$
Gallium citrate is absorbed by macrophages and reticular endothelial cells, and gallium scintigraphy has been shown to be $70 \%$ sensitive and $93 \%$ specific in diagnosing osteomyelitis. ${ }^{22}$ When combined with SPECT, it offers an even higher sensitivity and is the investigation of choice for monitoring response to treatment. ${ }^{17}$ However, the cost and the high radiation dose can limit its usage. ${ }^{17}$ In Case 1, although CT scan detected bony changes of temporal bone suggestive of SBO, it failed to accurately localize SBO. Subsequent nuclear medicine imaging localized SBO in the right greater sphenoid wing. In Case 2, signs of SBO were not detected on CT and MRI and the diagnosis was only made on nuclear medicine scanning. We suspect that cohort studies that used only CT and MRI in the diagnosis of SBO might have missed the diagnosis in some patients compared to if nuclear medicine imaging was employed..$^{13}$

These two cases highlight the importance of considering a diagnosis of SBO in non-diabetic elderly patients who present with even a mild chronic otalgia. This group of patients might not mount an adequate immune response as evident by lack of raised levels of inflammatory markers. Furthermore, a combination of CT and MRI might not be reliable in diagnosing or localizing SBO. We recommend nuclear medicine imaging should be part of diagnostic workup for accurate diagnosis and localization of SBO.

\section{Summary}

i. Otogenic skull base osteomyelitis can occur in non-immunocompromised patients without diabetes.

ii. Elderly patients with SBO can present with only mild otalgia and normal inflammatory markers.

iii. CT and MRI are useful in the workup, but might not be reliable in diagnosing or localizing SBO, as in Case 2 described here.

iv. Although not routinely done at present, nuclear medicine imaging should be part of diagnostic workup for accurate diagnosis and localization of SBO.

\section{Disclaimers}

Nothing to declare.

\section{Acknowledgements}

None.

\section{Conflict of interest}

Author declares that there is no conflict of interest.

\section{References}

1. Grandis JR, Yu VL. Malignant (necrotizing) external otitis. In: Post TW, editor. Waltham, USA; 2016.

2. Johnson AK, Batra PS. Central skull base osteomyelitis: an emerging clinical entity. Laryngoscope. 2014;124(5):1083-1087.

3. Lee S, Hooper R, Fuller A, et al. Otogenic cranial base osteomyelitis: a proposed prognosis-based system for disease classification. Otology \& neurotology: official publication of the American Otological Society. American Neurotology Society [and] European Academy of Otology and Neurotology. 2008;29(5):666-672.

4. Sreepada GS, Kwartler JA. Skull base osteomyelitis secondary to malignant otitis externa. Curr Opin Otolaryngol Head Neck Surg. 2003;11(5): 316-323. 
5. Chen CN, Chen YS, Yeh TH, et al. Outcomes of malignant external otitis: survival vs mortality. Acta oto-laryngol. 2010;130(1):89-94.

6. Leventhal D, Willcox TO, Evans JJ, et al. Bilateral skull base osteomyelitis in an immunocompetent patient. Ear Nose Throat J. 2011;90(12):E23E26.

7. Prasad KC, Prasad SC, Mouli N, et al. Osteomyelitis in the head and neck. Acta otolaryngol. 2007;127(2):194-205.

8. Gruver A, Hudson L, Sempowski G. Immunosenescence of ageing. $J \mathrm{~Pa}$ thol. 2007;211(2):144-156.

9. Geiger H, Rudolph KL. Aging in the lympho-hematopoietic stem cell compartment. Trends in immunology. 2009;30(7):360-365.

10. Ogawa T, Kitagawa M, Hirokawa K. Age-related changes of human bone marrow: a histometric estimation of proliferative cells, apoptotic cells, T cells, B cells and macrophages. Mech Ageing Dev. 2000;117(1-3):57-68

11. Blyth CC, Gomes L, Sorrell TC, et al. Skull-base osteomyelitis: fungal vs. bacterial infection. Clin Microbiol Infect. 2011;17(2):306-311.

12. Patmore H, Jebreel A, Uppal S, et al. Skull base infection presenting with multiple lower cranial nerve palsies. Am J Otolaryngol. 2010;31(5):376-380.

13. Ridder GJ, Breunig C, Kaminsky J, et al. Central skull base osteomyelitis: new insights and implications for diagnosis and treatment. European archives of oto-rhino-laryngology. 2015;272(5):1269-1276.
14. Kraus DH, Rehm SJ, Kinney SE. The evolving treatment of necrotizing external otitis. The Laryngoscope. 1988;98(9):934-939.

15. Stokkel MP, Boot CN, van Eck-Smit BL. SPECT gallium scintigraphy in malignant external otitis: initial staging and follow-up. Case reports. Laryngoscope. 1996;106(3 Pt 1):338-340.

16. Clark MP, Pretorius PM, Byren I, et al. Central or atypical skull base osteomyelitis: diagnosis and treatment. Skull Base. 2009;19(4):247-254.

17. Okpala NC, Siraj QH, Nilssen E, et al. Radiological and radionuclide investigation of malignant otitis externa. J Laryngol Otol. 2005;119(1):71-75.

18. Chaljub G, Van Fleet R, Guinto FC, et al. MR imaging of clival and paraclival lesions. AJR Am J Roentgenol. 1992;159(5):1069-1074.

19. Sharma P, Agarwal KK, Kumar S, et al. Utility of (99m)Tc-MDP hybrid SPECT-CT for diagnosis of skull base osteomyelitis: comparison with planar bone scintigraphy, SPECT, and CT. Jpn J Radiol. 2013;31(2):81-88

20. Adams A, Offiah C. Central skull base osteomyelitis as a complication of necrotizing otitis externa: Imaging findings, complications, and challenges of diagnosis. Clin Radiol. 2012;67(10):e7-e16.

21. Levin WJ, Shary JH, Nichols LT, et al. Bone scanning in severe external otitis. Laryngoscope. 1986;96(11):1193-1195.

22. Sorsdahl OA, Goodhart GL, Williams HT, et al. Quantitative bone gallium scintigraphy in osteomyelitis. Skeletal Radiol. 1993;22(4):239-242. 